\title{
Optimización Termoeconómica y Ambiental usando Algoritmos Genéticos Multiobjetivo
}

\author{
Marlon J. Bastidas ${ }^{(1)}$, Raúl F. Bermúdez ${ }^{(1)}$, Gloria P. Jaramillo ${ }^{(2)}$ y Farid Chejne ${ }^{(2)}$ \\ (1) Universidad Popular del Cesar (Grupo de Energías Alternativas y Biomasa), Salida a Patillal, \\ Valledupar-Colombia. \\ (2) Facultad de Minas-Universidad Nacional de Colombia, sede Medellín, Cra 80 N. ${ }^{\circ}$ 65-223, \\ Medellín-Colombia. (e-mail: mjbastid@unal.edu.co)
}

Recibido Nov. 05, 2009; Aceptado Dic. 21, 2009; Versión Final recibida Feb. 19, 2010

\begin{abstract}
Resumen
En este trabajo, se presenta un análisis multiobjetivo incluyendo variables del entorno de un sistema energético el cual es condicionado por el uso de combustibles sólidos, razón por la cual en el análisis se incluye la características externas del recurso (combustible), del residuo (cenizas) y del producto (electricidad/vapor). El sistema energético utilizado es Termocesar, un proceso colombiano de generación eléctrica que usa del carbón. Se analiza tres funciones denominadas función objetivo termoeconómica, función objetivo tecnológica y función objetivo ambiental, con el fin de determinar la mejor ubicación del sistema. Se usa el método de los algoritmos genéticos para la optimización del sistema. Los resultados fueron analizados para nueve combinaciones de pesos y muestran que para la mayoría de los casos el óptimo incluye carbones procedentes de la región del departamento del Cesar en Colombia.
\end{abstract}

Palabras Clave: algoritmos evolutivos, optimización, entorno, termo económico

\section{Thermoeconomic and Environment Optimization using Multiobjective Genetic Algorithms}

\begin{abstract}
This paper presents a multiobjective analysis including variables of the environment of the energy system which is conditioned by the use of solid fuels, for this reason the analysis includes the external characteristics of the resource (fuel) of residue (ash) and the product (electricity/steam). The energy system used is Termocesar, a Colombian process for generating electricity from carbon. Three functions named thermoeconomic objective function, technology objective function and environmental objective function, are analyzed to establish the best location of the energy The genetic algorithm method is used for the optimization of the system. The results were analyzed for nine combinations of weights and show that for most of the cases the optimum includes coals from the Department of Cesar in Colombia.
\end{abstract}

Keywords: evolutive algorithms, optimization, surrounding, thermoeconomic. 


\section{INTRODUCCIÓN}

La evolución de las máquinas térmicas, es propio del avance científico de las leyes físicas, debido a la necesidad de utilizar de mejor manera los recursos energéticos; cuyo fundamento conduce a desarrollar tecnologías más óptimas. La termodinámica, es una ciencia que lleva aportando importantes avances en el uso de la energía, desde el siglo XIX se han desarrollado diferentes modelos y aplicaciones, entre los que se pueden destacar el desarrollo de una herramienta para optimización dinámica, la evaluación de los parámetros de diseño en la optimización termodinámica en plantas de generación, la optimización en sistemas energéticos considerando los efectos ambientales contextualizados dentro de la termodinámica clásica. No obstante, los desarrollos teóricos de optimización de sistemas energéticos en los últimos años se demarcan en la exergoeconomía, teniendo en cuenta que los resultados obtenidos del análisis exergético considerando aspectos económicos, son más apropiados para la evaluación de un caso real que los obtenidos únicamente de un análisis energético (Tsatsaronis, 1993). La fusión de la termodinámica y le economía, ha permitido avanzar en la optimización de los recursos tanto económicos como energéticos. En los noventa se presentaron trabajos en el desarrollo de la metodología de optimización termoeconómica, lo que permitió constituirse como una teoría utilizada en diferentes estructuras energéticas, aunque el modelo base utilizado es el CGAM (Tsatsaronis y Pisa, 1994; Bejan et al., 1996).

Se han optimizado sistemas energéticos desde el punto de vista MO, generalmente conduciendo a óptimos locales, pero con la necesidad de utilizar varios criterios de evaluación para la escogencia de un sistema energético entre varias posibilidades (Garduno-Ramirez, 2001; Toffolo y Lazzaretto, 2002), teniendo en cuenta que la gestión, el diseño y planeamiento energético, considerando factores ambientales y económicos están en desarrollo (Pelet et al., 2005). No obstante, los trabajos sobre la predicción de contaminantes generados por sistemas energéticos y el mejoramiento de procesos para producir energía limpia a partir de productos de fácil obtención del entorno de dichos sistemas, están a la vanguardia (Lazaretto y Toffolo, 2008; Gassner y Maréchal, 2008). A pesar que el análisis MO carece de las herramientas para describir el funcionamiento físico y económico interno de un sistema energético, lo cual es propio de la termoeconomía; si tiene la posibilidad de evaluar sus condiciones óptimas de maniobrabilidad en un ambiente externo probable. El problema $\mathrm{MO}$ en sistemas energéticos complejos, cuando es evaluado con sistemas de cálculo convencionales, conduce a valores óptimos locales, por lo tanto se han utilizado herramientas de búsqueda para determinar diferentes posibilidades de escogencia entre varios óptimos o que conduzca a un óptimo global, como los algoritmos evolutivos (Sahoo, 2007; Salazar et al., 2006). Hasta ahora, las herramientas se han aplicado en la optimización de sistemas de conversión de energía, basada en los conceptos de la termodinámica y la economía incluyendo aspectos fundamentales para reducir el impacto ambiental (Meyer, et al., 2009); sin embargo, el análisis puede servir de complemento a la optimización MO, ya que permite incluir las variables externas a un sistema de energía complejo (Garduño et al., 2001), por lo que la teoría termoeconómica combinada con los algoritmos evolutivos, representa una herramienta muy poderosa para el estudio sistemático en la optimización de los sistemas energéticos (Sahoo, 2007; Toffolo y Lazzaretto, 2002). Este trabajo recoge algunos de los puntos de vista señalados anteriormente, pero además busca analizar un sistema energético de acuerdo a las características de entornos diferentes, para determinar según el criterio del decisor, un entorno favorable que sopese la maximización de los ingresos potenciales, la maximización del ahorro económico por utilizar una tecnología eficiente que contribuya a la producción limpia a través de la gasificación y la disminución del impacto ambiental por el uso de combustibles de calidad. Específicamente se compara Termocesar como sistema energético convencional y un sistema con las mismas características de mejor tecnología, con posibilidades de interactuación en diferentes escenarios colombianos como ubicación, tienendo en cuenta la calidad y costos de los combustibles (carbón) y la necesidad de utilizar mezclas de estos combustibles que satisfagan simultáneamente tres criterios de evaluación, que se dominan termoeconómico, tecnológico y ambiental.

\section{DESCRIPCIÓN DEL SISTEMA ENERGÉTICO PARA EL ANÁLISIS MULTIOBJETIVO}

Para describir el problema MO se parte de un sistema, como se muestra en la Figura 1. Este sistema, denominado Termocesar fue planeado teóricamente por entidades consultoras colombianas para 
generar $300 \mathrm{MW}$, como propuesta de generación eléctrica para suplir las crisis de verano en Colombia, con base en algunos requerimientos de entornos regionales, tales como la disponibilidad y calidad del carbón, el aprovisionamiento de agua de enfriamiento y condiciones adecuadas de vegetación para atenuar el impacto ambiental. El estudio reportó como ubicación propicia de Termocesar, al municipio de Tamalameque (Cesar- Colombia) por estar relativamente cerca de la zona carbonífera de donde se extraen carbones de buena calidad térmica, contar con abundantes bosques y estar contiguo a la cuenca del río Cesar como abastecedor permanente de agua. Las características principales de Termocesar, son el uso de la energía del carbón, el sistema con tres etapas de repotenciación y el agua de enfriamiento

El análisis incluye aspectos relacionados con las variables del entorno del sistema, esto con el fin de evaluar las condiciones del medio que afectan los recursos, los productos y los residuos, y a su vez evaluar como afectan estos al sistema de manera global. Por ejemplo, para el caso que el recurso sea carbón, habrá factores que condicionan el suministro, tales como la calidad, la disponibilidad, el precio, el tamaño y la ubicación del proveedor. De igual manera el precio, la calidad (electricidad/vapor) y la demanda son factores que condicionan el producto y la disposición final y/o el almacenamiento, condicionan los residuos. Se hace el análisis del sistema global, considerando tres aspectos de interés para evaluar: el desempeño termoeconómico, tecnológico y ambiental; lo que a su vez representan para el decisor, objetivos denominados con los mismos nombres. Un aspecto importante en el modelo, es incluir nuevas condiciones del entorno utilizando mezclas de carbones de diferentes regiones de Colombia; con esto se busca establecer si pueden emplearse otras regiones como factibles para la ubicación del sistema energético.

\section{Objetivo termoeconómico}

El análisis termoeconómico se ha centrado básicamente en establecer las implicaciones del costo exergético de las corrientes localizadas en sistema energético a través de los costos asociados al recurso y los equipos que lo conforman, salvo algunas excepciones metodológicas como las desarrolladas por Tsatsaronis (2005), han utilizado los ingresos para el análisis. Con el modelo presentado, a través del objetivo termoeconómico se puede inclusive, incluir los ingresos obtenidos en un ambiente de incertidumbre debido a las fluctuaciones del precio de la energía; aunque en este caso se propone hacer el ejercicio de certidumbre para esta variable. Con la inclusión del precio, se puede tener una aproximación de la capacidad real y teórica de ingresos, cuando el sistema está operando.

Para ello se considera que los ingresos teóricos, además de estar formados por el precio y la exergía producida, dependen del funcionamiento del sistema, es decir, de lo que se deja de deducir por el pago de la exergía perdida y destruida, por lo tanto los ingresos teóricos quedarían representados por la siguiente ecuación:

$\dot{I}_{T H}=p_{P} \dot{E}_{P}+c_{F}\left(\dot{E}_{L}+\dot{E}_{D}\right)$

Donde $\dot{E}_{P}$ es el flujo de exergía de la corriente de producto, $\dot{E}_{L}+\dot{E}_{D}$ es la suma de los flujos de exergía perdida y destruida, respectivamente; $p_{P}$ y $c_{F}$ son el precio y el costo exergético unitario del producto y del recurso, respectivamente. El flujo de ingresos $\left(\dot{I}_{T H}\right)$ depende directamente de la exergía producida y el precio de la exergía en el mercado; pero también dependen del estado del sistema, esto es, de la cantidad de exergía perdida y destruida debido a que sería el ingreso que se dejaría de recibir por el pago de la exergía no aprovechable.

Los ingresos reales, sería el producto por su precio en el mercado de la energía $\left(\dot{I}_{R}=p_{P} \dot{E}_{P}\right)$, con lo que el cociente del flujo de ingresos reales sobre el flujo de ingresos teóricos, denominamos como la "eficiencia potencial de ingresos" $\left(\varepsilon_{P I}=I_{R} / I_{T H}\right)$. La "eficiencia potencial de ingresos" se construye a partir del cociente de la exergía del producto y la exergía del recurso $\left(\varepsilon=\dot{E}_{P} / \dot{E}_{F}\right)$ y el balance de exegía del sistema, establecido como se muestra en la ecuación 2 (Sahoo, 2007).

$\dot{E}_{F}=\dot{E}_{P}+\dot{E}_{L}+\dot{E}_{D}$ 
De la ecuación 2, se puede obtener la relación de eficiencias con las exergías de las corrientes del sistema, construyéndose así la ecuación 3 (Tsatsaronis, 1993).

$\frac{1-\varepsilon}{\varepsilon}=\frac{E_{L}+E_{D}}{E_{P}}$

Tomando como referencia el concepto de eficiencia exergética y la ecuación 3, entonces el concepto de "eficiencia potencial de ingresos", se puede reescribir como aparece en la ecuación 4.

$\varepsilon_{P I}=\frac{1}{\left[1+\frac{c_{F}}{p_{P}}\left(\frac{1-\varepsilon}{\varepsilon}\right)\right]}$

Donde la eficiencia exergética es función del flujo de exergía del producto y la exergía contenida en la mezcla de combustibles que alimentan el sistema, tal como se muestra en la ecuación 5.

$\varepsilon=\frac{E_{P}}{\dot{m}_{T}\left(\sum X_{C B, i} \cdot P C_{i}\right)}$

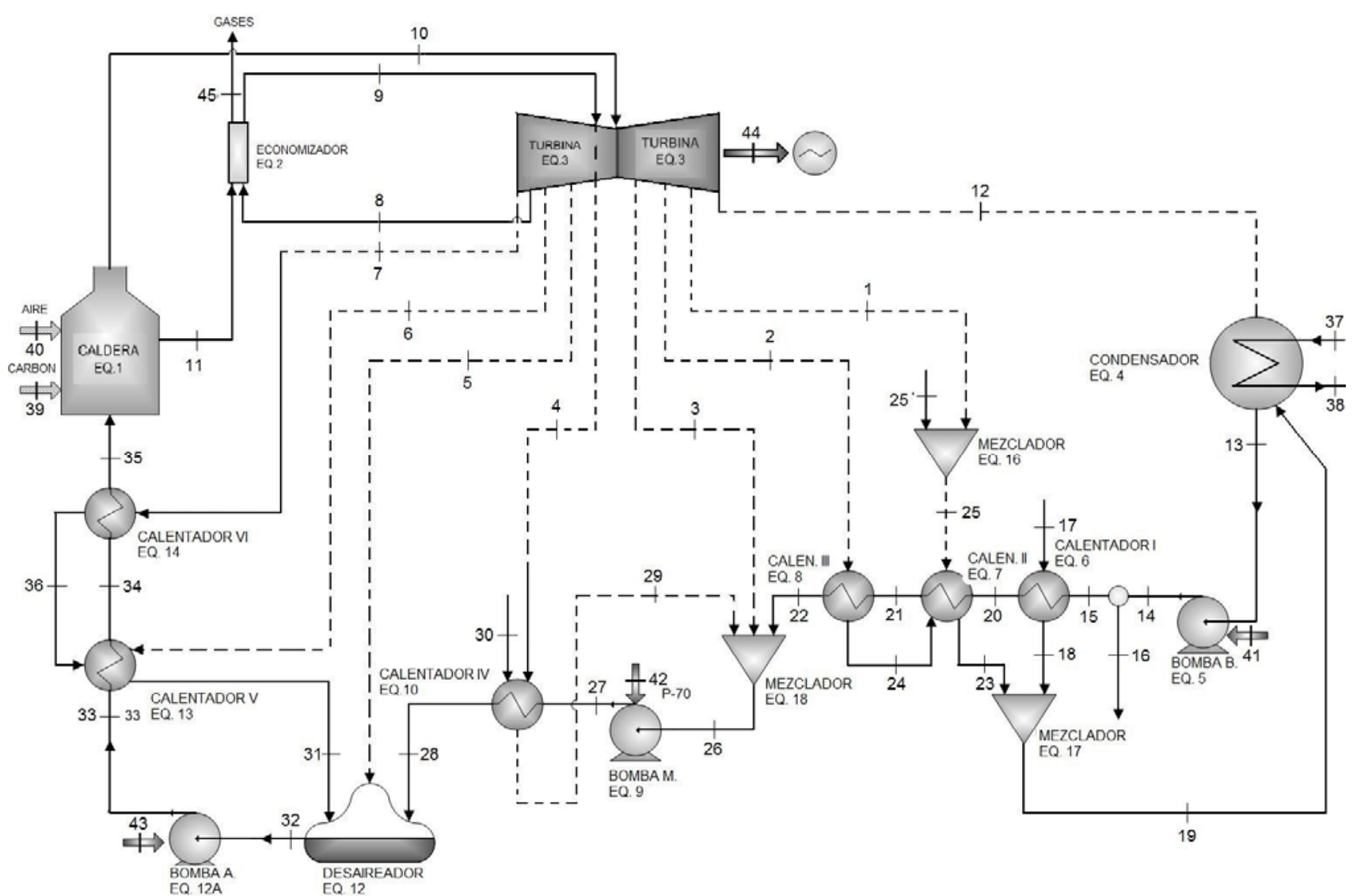

Fig. 1: Estructura de teórica de Termocesar para 300 MW

En este caso el objetivo sería maximizar la eficiencia potencial de ingresos $\left(\varepsilon_{P I}\right)$, con el que se quiere disminuir el costo debido a la exergía perdida y producida. Donde $\dot{m}_{T}$ es el flujo másico total de combustible y $\Sigma X_{C B}, P C_{i}$ es el poder calorífico de las fracciones de mezcla de combustibles. En este caso las fracciones de los combustibles son las variables de decisión. Las unidades de los flujos de exergía se dan en $\mathrm{kW}$, los flujos de ingresos en $U \$ / \mathrm{s}$, los costos exergéticos unitarios en $U \$ / \mathrm{kJ}$, el flujo másico en $\mathrm{kg} / \mathrm{s}$ y la eficiencia no tiene unidades. 


\section{Objetivo tecnológico}

Se parte del supuesto enunciado por Bejan et al., que dice que "para cada componente del sistema, se espera que los costos de inversión se aumenten con el aumento de la capacidad y el incremento de la eficiencia exergética del componente. De aquí, se asume que para el componente $k$ (o todo el sistema), el capital total de inversión $\left(T C l_{k}\right.$ ) puede ser representado en un mínimo aproximado dado por la siguiente relación:"

$T C I_{k}=B_{k}\left(\frac{\varepsilon_{k}}{1-\varepsilon_{k}}\right)^{n k} \dot{E}_{P, k}^{m k}$

La ecuación 6 es utilizada para optimización de sistemas energéticos a partir de criterios tecnológicos, tamaño o capacidad de los equipos y propiedades termodinámicas de las corrientes del sistema, la cual en conjunto con la ecuación 5 , conforman la ecuación 7 . La información económica utilizada en las estructuras, para determinar los valores de las constantes $B, n$ y $m$, se toman de información disponible del entorno, aplicando el método de mínimos cuadrados (Bejan et al., 1996).

La Fig. 1 se compara con otra estructura más eficiente, la Fig. 2, la cual se supone ocuparía el mismo entorno de Termocesar (mezclas de carbones de diferentes regiones de Colombia), produciendo 300 MW. La estructura utilizada en la Fig. 2, a pesar que en la actualidad no es una opción viable, es la referencia más cercana para evaluar la estructura de Termocesar, debido a que se mantienen las mismas condiciones de alimentación de combustible. Es claro, que una vez evaluado el sistema, un paso siguiente es proveer este sistema de un gasificador; por lo tanto se debe evaluar, si el aumento en la eficiencia obtenido en la estructura de la Fig. 2, genera el ahorro de dinero suficiente para la compra del gasificador y reemplazar con este la estructura de la Fig. 1.

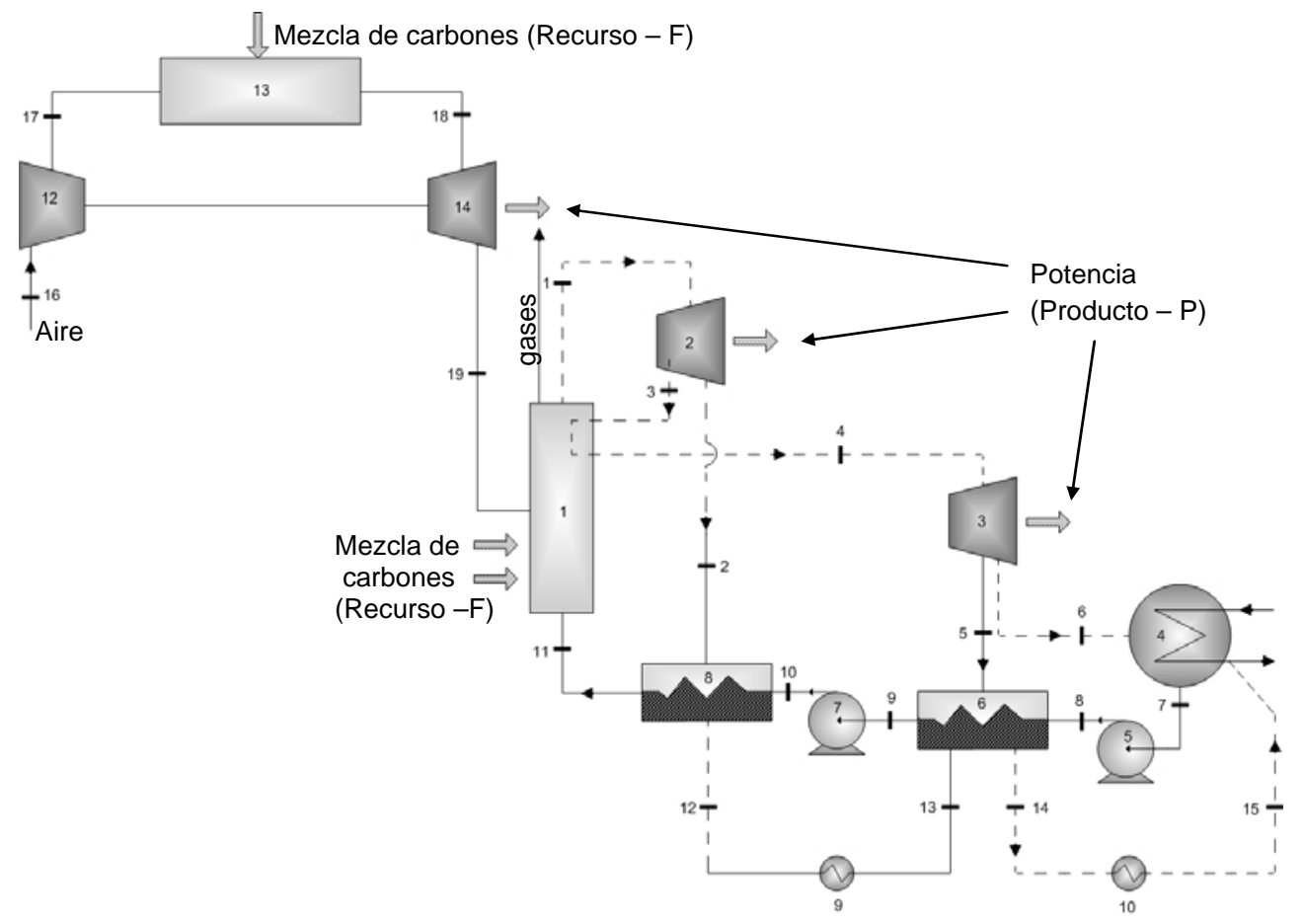

Fig. 2: Estructura de $300 \mathrm{MW}$ con mejora tecnológica

Teniendo las dos tecnologías, se puede establecer una diferencia de costos, con lo que se tiene el posible ahorro que permite utilizar una tecnología más eficiente que la convencional. El ahorro se puede representar con la ecuación 7.

$-A h^{A}=\tau \cdot C_{F}\left(\sum X_{C B, i} \cdot P C_{i}\right) \cdot\left(\dot{m}_{T}-\dot{m}_{T, o}\right)+\Delta C_{C O}+\Delta C_{O \& M}$ 
El primer término corresponde a la diferencia de cantidades de combustibles utilizados entre los dos sistemas. Los dos últimos factores de la ecuación 7, corresponde a la diferencia de los costos debido a las condiciones de operación del sistema energético $\left(\Delta C_{C O}\right)$ y la diferencia debido a los costos de operación y mantenimiento $\left(\Delta C_{O \& M}\right)$. La primera relación $\left(\Delta C_{C O}\right)$ se obtiene de la ecuación 6 (función del capital total invertido), la segunda relación $\left(\Delta C_{O \& M}\right)$ hace referencia a los costos de operación y mantenimiento variable y otros costos remanentes. Las unidades de ahorro y diferencia de costos tanto en los combustibles, las condiciones de operación como en la operación y mantenimiento del sistema se da en U\$ para un año.

$$
\begin{aligned}
& \Delta C_{C O}=E_{P}^{m} \cdot(\beta+\gamma) \cdot B\left[\frac{E_{P}}{\dot{m}_{T}\left(\sum X_{C B, i} \cdot P C_{i}\right)-E_{P}}\right]^{n}-E_{P}^{m, o} \cdot\left(\beta_{o}+\gamma_{o}\right) \cdot B_{o}\left[\frac{E_{P}}{\dot{m}_{T, o}\left(\sum X_{C B, i} \cdot P C_{i}\right)-E_{P}}\right]^{n, o}, \mathrm{y} \\
& \Delta C_{O \& M}=\tau \cdot \dot{E}_{P} \cdot\left(\varpi-\varpi_{o}\right)+\left(R-R_{o}\right)
\end{aligned}
$$

Donde el subíndice $o$, indica los valores de la tecnología convencional, $\tau$ (8000 horas) es el tiempo de operación en horas durante un año (superíndice $A$ ). Las letras $\beta, \gamma, \varpi$ y $R$ corresponden a los costos de operación y mantenimiento constantes, el factor de recuperación, los costos de operación y mantenimiento variables y otros costos, respectivamente que dependen del capital invertido.

Los valores de las constantes $B, n$ y $m$ tanto para el sistema convencional (SC) como para el sistema optimizado (SO), se muestran en la tabla 1 y se obtienen asumiendo uno o dos valores de las constantes, dependiendo de la información disponible, y se calculan los restantes por el método de los mínimos cuadrados en la ecuación 6 (Tsatsaronis, 1993; Bejan et al., 1996). Para los valores de los parámetros, AENE (1997) para el SC y Bermúdez (2005) para el SO, reportaron los siguientes valores respectivamente: SC (300 MW de capacidad, equipos e instalación ascienden a US\$1259.97 por cada kW instalado; y eficiencia neta de 38.3\%); SO (costo de equipos e instalación son de US\$ 1475.4 por cada kW instalado, $300 \mathrm{MW}$ de capacidad y eficiencia neta de 40.6\%). Para ambos casos se utilizó un tiempo de vida útil de la planta de 20 años y $p_{P}$ promedio de U\$ 40/MWh.

Tabla 1: Constantes de la ecuación 6 para SC y SO

\begin{tabular}{|c|c|c|c|c|c|c|c|c|c|c|}
\hline Parámetros & $\beta$ & $\beta_{o}$ & $V$ & $V_{o}$ & $B$ & $B_{o}$ & $m$ & $m_{o}$ & $n$ & $n_{o}$ \\
\hline Valor & 0.182 & \multicolumn{2}{|c|}{1.092} & $19.2 \mathrm{E} 3$ & 5.27 & 0.47 & 1.06 & 0.29 & 0.32 \\
\hline
\end{tabular}

Los datos faltantes para completar la solución de la ecuación 7, corresponden a los costos y calidades de las variables de decisión constituidas por la fracción de cada uno de los carbones que hacen parte de una mezcla que alimenta el sistema energético. Los carbones colombianos presentan diferentes calidades y precios, tal como se aprecia en la tabla 2, lo que hace interesante encontrar una mezcla óptima de estos carbones. En la tabla 2, se aprecia que los carbones de mayor poder calorífico proceden de las regiones de Santander, Cesar, Guajira, Antioquia y Boyacá; pero sólo en los carbones del Cesar y la Guajira, el contenido de cenizas es inferior al 9\%, lo que hace que su costo sea alto comparado a los demás, entendiendo que estos carbones sean apetecibles en el mercado internacional.

Tabla 2: Carbones de diferentes regiones de Colombia

\begin{tabular}{|c|c|c|c|c|}
\hline No. & $\begin{array}{c}\text { Lugar de origen del } \\
\text { carbón }\end{array}$ & $\begin{array}{c}\mathrm{PC} \\
(\mathrm{kJ} / \mathrm{kg})\end{array}$ & $\begin{array}{c}\text { Contenido } \\
\text { de cenizas } \\
(\%)\end{array}$ & $\begin{array}{c}\text { Price 2008 } \\
\mathbf{W} / 1000 \\
\mathrm{~kg}\end{array}$ \\
\hline 1 & Antioquia y Caldas & 28482.75 & 9.12 & 37.19 \\
\hline 2 & Valle del Cauca & 22436.64 & 30.40 & 28.30 \\
\hline 3 & Boyacá & 28011.32 & 11.55 & 26.42 \\
\hline 4 & Cundinamarca & 27408.44 & 10.19 & 26.80 \\
\hline 5 & Cesar & 28759.25 & 5.28 & 46.73 \\
\hline 6 & Guajira (Cerrejón) & 28833.62 & 8.30 & 47.18 \\
\hline 7 & Córdoba & 25328.72 & 17.00 & 25.73 \\
\hline 8 & Santander & 30260.67 & 16.35 & 44.88 \\
\hline
\end{tabular}




\section{Objetivo ambiental}

Se establece como la cantidad másica de residuos sólidos o cenizas $\left(m_{z}\right)$ que se generan por la combustión del carbón, tal como se aprecia en la ecuación 8. En este caso se toma la fracción de cenizas $\left(Y_{i}\right)$ que contiene cada combustible, debido a que esta tiene incidencia directa con la calidad de dichos combustibles, aunque para casos de análisis globales se puede usar indicadores como el CDE de reducción en emisiones de dióxido de carbono (Mago y Chamra, 2009).

$m_{Z}^{A}=\tau \cdot \dot{m}_{T}\left(\sum X_{C B, i} \cdot Y_{i}\right)$

Con esta función, se tiene la certeza que la evaluación se enfoca en la escogencia del tipo de combustible, mientras que se evalúa la eficiencia del sistema a través de las funciones objetivo termoeconómica y tecnológica. Si se utilizan indicadores ambientales globales, es posible que la función objetivo tenga aspectos parecidos con los objetivos anteriores y se pierda precisión en la meta esperada, la cual es la escogencia del mejor combustible para el escenario planteado.

\section{METODOLOGIA DE EVALUACION APLICANDO ALGORITMOS EVOLUTIVOS}

La presencia de múltiples objetivos en un problema, además de requerir de un método de resolución no convencional, da lugar a un grupo de soluciones óptimas conocidas ampliamente como soluciones óptimas de Pareto.

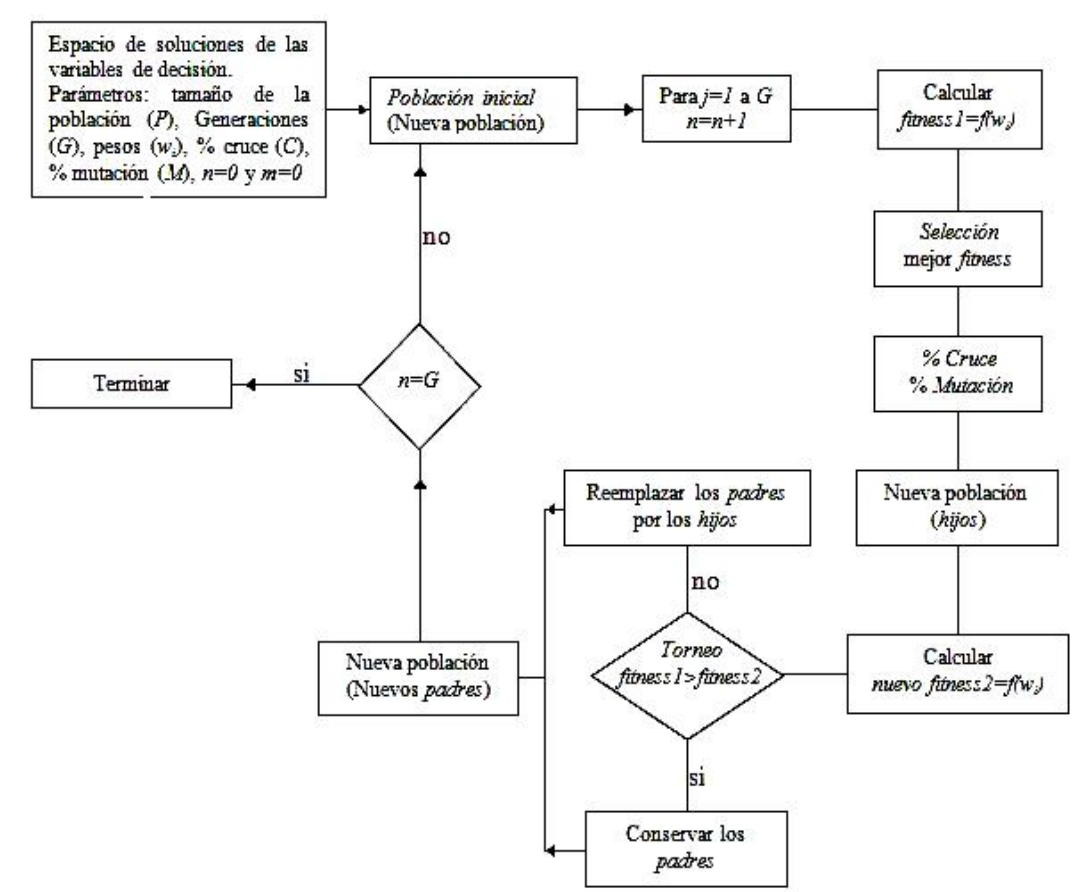

Fig. 3: Diagrama de flujo del AG aplicando ponderación lineal

Los métodos evolutivos como la programación evolutiva, las estrategias evolutivas y los algoritmos genéticos (AG) son los más utilizados para estos casos debido a que tienen la ventaja de aplicar en modelos de alta complejidad que difícilmente tienen solución con metodologías matemáticas convencionales, porque pueden evaluar infinitas soluciones óptimas. En lo particular empleamos AG, con el que se construye el grupo de soluciones óptimas a partir de la asignación de diferentes conjuntos de pesos de importancia para cada función objetivo. En la Fig. 3, se muestra el diagrama de flujo del algoritmo genético por pesos, en el que se identifican cada paso requerido para que se alcance la optimización para el caso particular. Los AG's son una representación simple de la evolución biológica, en la que un par de padres de una población de individuos demarcados en una generación (selección), dan origen a un par de hijos con características de los padres (cruzamiento). Los individuos que forman la próxima generación se escogen por torneo, en el que gana el que tenga mejor aptitud (fitness-ecuación 9) con base en los criterios del evaluador, con lo que la optimización 
va evolucionando hacia mejores valores en las próximas generaciones. Antes de la evaluación del fitness, se estima un porcentaje pequeño de mutación con el que hay un probable cambio de alguna característica del individuo.

\section{RESULTADOS Y DISCUSIÓN}

Queda definido un problema MO, en el que el decisor pretende maximizar el objetivo termoeconómico y el tecnológico, pero minimizando el ambiental; para lo que requiere de técnicas no convencionales de optimización (Padilha et al., 2009). Para este caso se empleó la técnica de algoritmos evolutivos, desarrollando la frontera de Pareto asignándole peso a cada uno de los objetivos (método de ponderación lineal). Los pesos ( $w$ ) son distribuidos a cada objetivo obteniendo una función de aptitud global $(F A)$, que se utiliza para la optimización, tal como se muestra en la ecuación 9.

$\operatorname{Max}: F A=\varepsilon_{P I} \cdot w_{1}+(-A h) \cdot w_{2}-m_{z} \cdot w_{3}$

Donde $w_{1}+w_{2}+w_{3}=1$. Como se requiere minimizar $m_{z}$, entonces se maximiza el negativo. Inicialmente se hace una exploración de la cantidad de individuos y generaciones necesarios para encontrar mejores valores. En la Fig. 3, se muestra el progreso de búsqueda del algoritmo evolutivo empleando diferente cantidad de individuos y generaciones para un problema MO integrado en uno solo, con pesos iguales para cada objetivo.

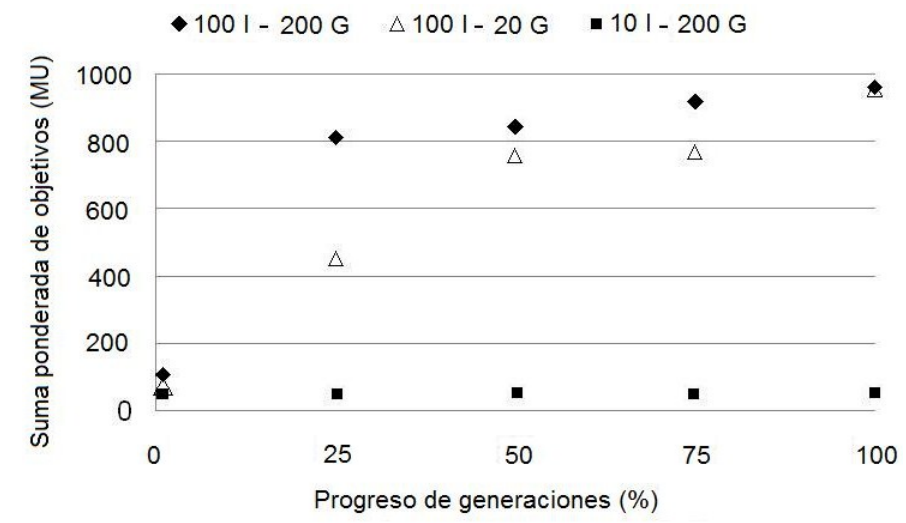

Fig. 3. Optimización evolutiva para diferentes individuos y generaciones

Cuando se tiene la menor cantidad de individuos ( $I$ ) no alcanza buenos valores, esto se debe a que no hay suficiente espacio de búsqueda para encontrar mejores combinaciones. Los puntos para más individuos tienen mejores resultados, pero la de mayor número de generaciones $(G)$, mayores valores de la FA. Se eligieron como mejores parámetros 100 individuos y 200 generaciones para nueve ensayos a diferentes pesos. Los resultados para cada ensayo se muestran en la tabla 3, en la que están consignados tres grupos de valores así: los primeros corresponden a los pesos ( $w_{1}$ :termoeconómico, $w_{2}$ :tecnológico y $w_{3}$ :ambiental) asignados a cada función objetivo; los segundos corresponden a la fracción de combustible $(X)$ conformado por carbones de diferentes regiones de Colombia, para los nueve conjunto de pesos; y los terceros corresponden a los valores óptimos de las funciones objetivo $\left(\varepsilon_{P l},-A h, m_{z}\right)$ para cada conjunto de pesos. La tabla 3 , muestra la influencia del objetivo ambiental en los otros objetivos y la predominancia del objetivo tecnológico. Cuando se le asigna un peso alto al objetivo ambiental, se observa que los objetivos termoeconómico y tecnológico se afectan, en algunos casos considerablemente. Cuando el objetivo ambiental se le da un peso de 0.5 (ensayos 1, 3 y 8), sólo hay una respuesta positiva del ahorro cuando el objetivo termoeconómico tiene un peso alto (0.4), sin embargo, el porcentaje de "eficiencia potencial de ingresos" en estas condiciones es muy pequeña (0.3\%) - ensayo 3; caso contrario ocurre cuando se asigna peso alto al objetivo tecnológico (0.4), generando un ahorro pequeño (12.8\%) - ensayo 1 . Cuando se tiene el mayor porcentaje de "eficiencia potencial de ingresos" (100\%), también se genera la mayor cantidad de residuos (100\%) y el ahorro con respecto al sistema convencional es cero (ensayo 5). Los mayores ahorros frente a la tecnología convencional se alcanzan cuando se utiliza en mayor proporción carbones del Cesar $\left(X_{5}\right)$, como se muestra en los ensayos $3(99.8 \%)$ y $9(100 \%)$, ensayos en los que se logra también los menores porcentajes de residuos ( $1 \%$ y $0 \%$, respectivamente). Para 
obtener valores altos en la "eficiencia potencial de ingresos", se nota que debe haber una mezcla de combustibles entre los que siempre está en mayor proporción el carbón del Cesar, pero intervienen otros carbones como el de la Guajira $\left(X_{6}\right)$ y Boyacá $\left(X_{3}\right)$. Los carbones que definitivamente no alcanzan representatividad para ser utilizados en la generación del sistema energético de $300 \mathrm{MW}$, son los carbones de Antioquia y caldas $\left(X_{1}\right)$ y Valle del Cauca $\left(X_{2}\right)$, los cuales no superaron valores de 0.147 en las mezclas óptimas.

Tabla 3: Ensayos del algoritmo evolutivo para diferentes pesos

\begin{tabular}{|c|c|c|c|c|c|c|c|c|c|}
\hline Ensayos óptimos & 1 & 2 & 3 & 4 & 5 & 6 & 7 & 8 & 9 \\
\hline Pesos & $w_{1}=0.1$ & $w_{1}=0.1$ & $w_{1}=0.4$ & $w_{1}=0.4$ & $w_{1}=0.5$ & $w_{1}=0.5$ & $w_{1}=.25$ & $w_{1}=.25$ & $w_{1}=.33$ \\
& $w_{2}=0.4$ & $w_{2}=0.5$ & $w_{2}=0.1$ & $w_{2}=0.5$ & $w_{2}=0.4$ & $w_{2}=.25$ & $w_{2}=0.5$ & $w_{2}=.25$ & $w_{2}=.33$ \\
Composición & $w_{3}=0.5$ & $w_{3}=0.4$ & $w_{3}=0.5$ & $w_{3}=0.1$ & $w_{3}=0.1$ & $w_{3}=.25$ & $w_{3}=.25$ & $w_{3}=0.5$ & $w_{3}=.33$ \\
\hline$X_{1}$ & 0.034 & 0.000 & 0.002 & 0.230 & 0.147 & 0.000 & 0.004 & 0.002 & 0.000 \\
\hline$X_{2}$ & 0.000 & 0.000 & 0.002 & 0.000 & 0.000 & 0.001 & 0.000 & 0.000 & 0.000 \\
\hline$X_{3}$ & 0.141 & 0.001 & 0.000 & 0.187 & 0.145 & 0.000 & 0.000 & 0.000 & 0.001 \\
\hline$X_{4}$ & 0.141 & 0.000 & 0.000 & 0.028 & 0.145 & 0.001 & 0.005 & 0.000 & 0.000 \\
\hline$X_{5}$ & 0.278 & 0.515 & 0.995 & 0.301 & 0.170 & 0.436 & 0.494 & 0.561 & 0.996 \\
\hline$X_{6}$ & 0.124 & 0.482 & 0.000 & 0.066 & 0.101 & 0.562 & 0.491 & 0.436 & 0.002 \\
\hline$X_{7}$ & 0.141 & 0.001 & 0.001 & 0.000 & 0.145 & 0.000 & 0.003 & 0.000 & 0.000 \\
\hline$X_{8}$ & 0.141 & 0.001 & 0.000 & 0.187 & 0.145 & 0.000 & 0.002 & 0.000 & 0.000 \\
\hline$\varepsilon_{P I}(\%)$ & 87.2 & 64.0 & 0.3 & 91.9 & $\mathbf{1 0 0 . 0}$ & 74.4 & 66.2 & 57.5 & 0.0 \\
\hline$-A h(\%)$ & 12.8 & 85.9 & 99.8 & 28.5 & 0.0 & 84.1 & 84.0 & 87.0 & $\mathbf{1 0 0 . 0}$ \\
\hline$m_{z}(\%)$ & 91.5 & 25.5 & 1.0 & 77.0 & 100.0 & 29.8 & 27.0 & 22.8 & $\mathbf{0 . 0}$ \\
\hline
\end{tabular}

\section{CONCLUSIONES}

La optimización de sistemas energéticos complejos, no puede ser exclusivo del sistema interno. El entorno es importante para definir un sistema energético adecuado amparado en los diferentes factores que ejercen influencia para decidir sobre el óptimo. Con el uso de algoritmos genéticos multiobjetivo (AGMO) en la optimización, fue posible obtener las siguientes conclusiones:

$\checkmark \quad$ Los factores del entorno intervienen en la optimización del sistema energético y le permite al decisor manejar una información general del sistema, en el que puede hacer inferencia tanto en las condiciones del sistema energético, como de los recursos que tiene a sus alrededores. Tener un sistema muy eficiente, no significa que sea el óptimo, debido a que el entorno condiciona esta premisa. En este sentido, si el decisor requiere establecer la ubicación de un sistema para generar los $300 \mathrm{MW}$ bajo los escenarios presentados por las calidades de los carbones y las funciones objetivo planteadas en este trabajo, sería los carbones del Cesar la mejor opción presentada con el análisis, esto se nota porque en la mayoría de los nueve conjuntos de pesos, la fracción del combustible de esta región de Colombia es la más alta. Faltaría determinar un único conjunto de pesos que definan las condiciones óptimas sistema-alrededores, que sería objeto de estudio en otra oportunidad.

$\checkmark \quad$ El objetivo ambiental, que generalmente es incluido en el objetivo termoeconómico (minimización de costos), ejerce una marcada influencia sobre los demás objetivos; lo cual se nota al asignarle valores altos a este objetivo.

$\checkmark \quad$ Para pesos altos en el objetivo ambiental, las variables de decisión optan por tomar carbones de bajo contenido de cenizas en el proceso de optimización, aunque tenga precios por encima de los demás. Lo importante de esta metodología, es que se pueden obtener mezclas óptimas de los combustibles que se utilizan, en concordancia con los requerimientos tecnológicos y la disponibilidad de recursos para hacer la inversión y las necesidades energéticas del entorno.

\section{AGRADECIMIENTOS}

Los investigadores agradecen el apoyo a COLCIENCIAS por la financiación de la beca doctoral a Marlon Bastidas Barranco y al doctor Blás Galván director del CEANI de la ULPGC. 


\section{REFERENCIAS}

AENE Consultoría S.A., Servicios de consultoría técnica, económica, legal y ambiental, Bogotá, Colombia (1997).

Bermúdez M. "Evaluación exergoeconomica de Termocesar en la ZEEE”, Informe de Investigación, Universidad Popular del Cesar (2005).

Bejan, A., Tsatsaronis, G. y Moran, M., "Thermal Design and Optimization”, Jhon Wiley and Sons, Inc. New York, 463-513 (1996).

Cziesla. F., Tsatsaronis, G. y Gao. Z, "Avoidable thermodynamic inefficiencies and costs in an externally fired combined cycle power plant", Energy, Vol. 31, 1472-1489 (2006).

Mago P. y Chamra L., "Analysis and optimization of CCHP systems based on energy, economical, and environmental considerations", Energy and Buildings (2009).

Garduno-Ramirez, R. y Lee, K.Y., "Multiobjective optimal power plant operation through coordinate control with pressure Set point scheduling", IEEE Transactions on Energy Conversion, Vol. 16, No. 2, 115-122, (2001).

Gassner M. y Maréchal F, "Thermo-economic optimisation of the integration of electrolysis in synthetic natural gas production from wood", Energy, Vol. 33, 189-198 (2008).

Lazzaretto A. y Toffolo A., "Prediction of performance and emissions of a two-shaft gas turbine from experimental data", Applied Thermal Engineering, Vol. 28, 2405-2415 (2008).

Lozano, A y Valero, A., "Theory of the Exergetic Cost”, Energy, Vol. 18, No. 9, 939 - 960 (1993).

Meyer L., Tsatsaronis G., Buchgeister J., Schebek L., "Exergoenvironmental analysis for evaluation of the environmental impact of energy conversion systems". Energy. No. 34 , 75-89 (2009).

Padilha, R.S., Santos, H.F.L., Colaco, M.J., Cruz, M.E., "Single and Multi-Objective Optimization of a Cogeneration System Using Hybrid Algorithms". Heat Transfer Engineering, vol. 30, 261-271 (2009).

Pelet X., Favrat D., Leyland G., Multiobjective optimisation of integrated energy systems for remote communities considering economics and CO2 emissions., International Journal of Thermal Sciences., Vol. 44, pp. 1180-1189 (2005).

Sahoo P. K., "Exergoeconomic analysis and optimization of a cogeneration system using evolutionary programming", Applied thermal engineering, Volume 28, Issue 13, 1580-1588 (2007).

Salazar, D., Rocco, C. M. y Galván B., "Optimization of constrained multiple-objective reliability problems using evolutionary algorithms", Reliability Engineering \& System Safety, Volume 91, Issue 9, 1057-1070 (2006).

Toffolo A. y Lazzaretto A., Evolutionary algorithms for multi-objective energetic and economic optimization in thermal system design, Energy., Vol. 27., pp. 549-567 (2002).

Tsatsaronis, G, "Thermoeconomic analysis and optimization of energy systems". Progress in energy and combustion science, Vol. 19, No 3, 227-257 (1993).

Tsatsaronis, G. y Pisa, J., "Exergoeconomic evaluation and optimization of energy systems Application to the CGAM problem", Energy, Vol. 19, No. 3, pp. 287-321 (1994). 